\title{
Un lectionnaire nestorien des évangiles selon l'ordo de Mossoul, écrit en juin 1706
}

A Nestorian Lectionary of the Gospels according to the Ordo of Mosul, Written in June 1716

$$
\text { ليتورجيا طقسية نسطورية للأناجيل بحسب أخوية الموصل كتبت سنة IVI }
$$

\section{Alain Desreumaux}

\section{OpenEdition}

\section{Journals}

Édition électronique

URL : http://journals.openedition.org/beo/544

DOI : $10.4000 /$ beo.544

ISBN : 978-2-35159-335-6

ISSN : 2077-4079

Éditeur

Presses de l'Institut français du Proche-Orient

Édition imprimée

Date de publication : 1 mai 2012

Pagination : 289-313

ISBN : 978-2-35159-193-2

ISSN : 0253-1623

\section{Référence électronique}

Alain Desreumaux, «Un lectionnaire nestorien des évangiles selon l'ordo de Mossoul, écrit en juin

1706 », Bulletin d'études orientales [En ligne], Tome LX | mai 2012, mis en ligne le 31 mai 2012, consulté le 19 avril 2019. URL : http://journals.openedition.org/beo/544 ; DOI : 10.4000/beo.544 
UN LECTIONNAIRE NESTORIEN DES ÉVANGILES SELON L'ORDO DE MOSSOUL, ÉCRIT EN JUIN 1716

\author{
Alain DesReumaux, \\ Directeur de recherche au CNRS \\ (Orient \& Méditerranée, Études sémitiques)
}

Les livres liturgiques constituent une part considérable du patrimoine culturel des communautés chrétiennes. Dans les Églises syriaques, ils jouent un rôle essentiel pour le déroulement des célébrations liturgiques et les idées théologiques et spirituelles véhiculées par les hymnes et prières diverses ${ }^{1}$. Rédigés sous les directives des autorités patriarcales et épiscopales, ils ont été multipliés par les mains d'innombrables copistes et relieurs. Ces manuscrits sont les témoins des pratiques officielles des grandes Églises et aussi les reflets de pratiques locales; ils sont donc de précieux documents pour les historiens.

L'Église syro-orientale utilise plusieurs livres liturgiques. Le hudrā, littéralement " cycle », contient les prières des heures avec leurs hymnes, repons et antiennes de tous les dimanches de l'année liturgique; le kaškūl contient les prières des heures pour les jours de la semaine ; le daqdām wadbätar, littéralement « Avant et après » est le recueil de prières et de strophes psalmiques récitées les matins et les soirs de la semaine ; l'ensemble constitue un recueil de grande ampleur; l'édition de celui de la métropolie nestorienne de l'Inde ${ }^{2}$ comprend trois gros volumes de quatre cent soixante-quinze pages chacun. Le rituel de la messe est appelé quddāšă, littéralement « consécration ». Les lectures des textes évangéliques du cycle liturgique sont rassemblées dans des lectionnaires qui suivent le calendrier de l'année ; une péricope précise est attribuée à chaque dimanche (et souvent aux samedi et même aux vendredi), ainsi qu'aux fêtes dites « de Notre-Seigneur » (Nativité, Épiphanie, etc.) et à quelques fêtes du sanctoral (Pierre et Paul, Georges...) ; les lectures des épîtres pauliniennes sont rassemblées dans le lectionnaire appelé slịhā, littéralement «l'Apôtre ». Le recueil des turgāme, littéralement « commentaires », contient ce que chez les latins on appellerait sequentiae, hymnes chantés entre l'épître et l'évangile à la messe. Le gazzā est un recueil d'hymnes pour les offices des dimanches et fêtes. Le rituel des défunts fait l'objet d'un volume particulier appelé 'annīdā.

1. Sur les liturgies syriaques en général, on consultera l'ouvrage réalisé dans le cadre de la Société d'études syriaques par CASSINGENA-TRÉVEDY \& JuRAsZ, 2006.

2. DARMo, 1960, 1993². Là encore, l'ordo est celui du couvent Mar-Gabriel-et-Mar-Abraham de Mossoul. 
Ces recueils ont naturellement évolué au cours des siècles, traduisant les grandes réformes liturgiques successives opérées par plusieurs grandes figures patriarcales: Ǐšóyahb III (649-659), Simon VI (1504-1538) notamment. Toutefois, la référence liturgique de l'Église syro-orientale demeure le rite du couvent Mar-Gabriel-et-Mar-Abraham de Mossoul, jusqu'au $x^{\mathrm{e}}$ siècle. Le lectionnaire qui fait l'objet de la présente étude se situe explicitement dans cette tradition; il appartient à la série des livres liturgiques postérieurs à la réforme de Simon VI.

Il s'agit d'un manuscrit en papier $(320 \times 218 \times 22 \mathrm{~mm})$ de 112 folios, en écriture syroorientale, aujourd'hui conservé dans la communauté assyro-chaldéenne de la région parisienne. C'est madame Catherine Treccani, directrice de l'atelier de reliure Ars Libris à Écouen, qui a attiré mon attention sur ce livre et à qui je suis très reconnaissant de m'avoir permis de l'examiner. Véronique Desreumaux en a dessiné la reliure d'origine et a participé à sa description codicologique.

Nous pensons que la description exhaustive de ce manuscrit soigneusement mis en pages et confectionné au début du xvIII ${ }^{\mathrm{e}}$ siècle peut contribuer à l'histoire de la liturgie syro-orientale et à celle de la codicologie syriaque, d'autant plus que fort peu de manuscrits nestoriens ont été conservés jusqu'à aujourd'hui ${ }^{3}$. Il a en effet été écrit en juin 1716 ap. J.-C. au temps du patriarche nestorien de Qočanes, mār Šem'ōn XIV Salomon à l'église Mar-Giwargīs de B-Lāhēh ${ }^{4}$, dans la région d'Anzal, à l'ouest d'Urmia, dans le Kurdistan iranien, pour les besoins de cette église inconnue par ailleurs ${ }^{5}$.

C'est pourquoi nous en faisons hommage à Mgr Boutros Yousif et à la communauté chaldéenne originaire d'Iraq et de Turquie résidant aujourd'hui dans notre pays.

\section{A. Hudra des dimanches et fêtes (f. $1^{\mathrm{va}}-109^{\mathrm{va}}$ )}

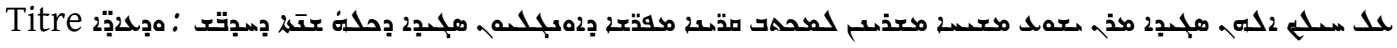

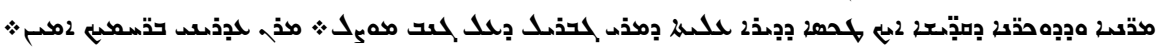

- 1. Premier dimanche de l'Annonciation (f. $1^{\mathrm{va}}-3^{\mathrm{rb}}$ )

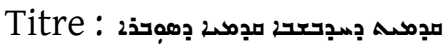

Antienne

Texte : Lc 1, 1-25.

- 2. Premier vendredi de l'Annonciation : c'est la Commémoraison de mar Babai de Nisibe, de Jean et de tous leurs compagnons (f. $3^{\mathrm{rb}}$ )

3. Amir Harrak, professeur à l'Université de Toronto, prépare la publication d'un catalogue de manuscrits de Bagdad dans lequel il souligne ce fait. L'apport de son catalogue détaillé et érudit est d'un grand intérêt, tant pour les textes que pour la codicologie et la paléographie. Je suis particulièrement reconnaissant à Amir Harrak de m'avoir communiqué ce travail en cours et de m'avoir autorisé à le citer pour compléter les informations du présent article dans lequel toutes les références aux manuscrits de Bagdad renvoient à son ouvrage; en outre ses corrections et observations m'ont été d'un grand secours.

4. On peut supposer que ce nom s'explique étymologiquement par la forme Bēt-Lāhēh: cette dérivation toponymique est connue dans les régions syro-orientales.

5. Le village de B-Lāhēh ne figure pas dans les listes (p. 329 et 331) du district de Anzel (p. 328-332) étudié par WILMSHURST, 2000. 


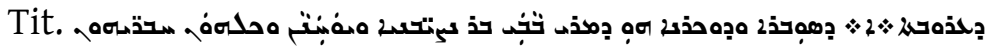

Aucun texte n'est indiqué.

- 3. Deuxième dimanche de l'Annonciation (f. $3^{\mathrm{rb}}-4^{\mathrm{vb}}$ )

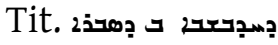

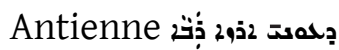

Texte : Lc 1, 26-56.

- 4. Troisième dimanche de l'Annonciation (f. $5^{\mathrm{ra}}-6^{\mathrm{ra}}$ )

Tit.

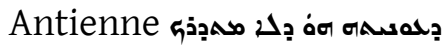

Texte : Lc 1, 57-80.

- 5. Quatrième dimanche de l'Annonciation (f. $6^{\mathrm{ra}}-6^{\mathrm{vb}}$ )

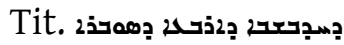

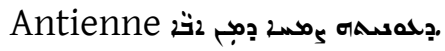

Texte : $M t 1,18-25$.

- 6. Fête de la Nativité de Notre-Seigneur (f. $6^{\mathrm{vb}}[$ )

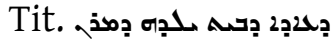

Il ne reste que le premier mot de la lecture.

Texte : Lc 2, 1[-20] ; d'après le ms. Cambridge, Add. 1975, on est en droit de restituer cette péricope puisque le titre et le premier mot correspondent.

lacune de deux folios qui contenaient tout le texte et le début du texte suivant.

- 7. [Premier dimanche après la Nativité] (f. ] $\left.7^{\mathrm{ra}}-7^{\mathrm{va}}\right)$

Tit. manque.

Texte : Mt 2, [1-12]13-20a.22-23.

On peut restituer le début de cette péricope d'après le ms. Cambridge, Add. 1975.

- 8. Commémoraison de Mart Mariam (f. $7^{\mathrm{va}}$ )

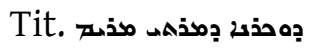

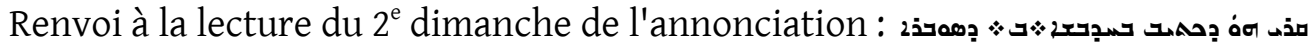
c'est-à-dire $L c$ 1, 26-56.

- 9. Commémoraison de l'été (f. $\left.7^{\mathrm{va}}-8^{\mathrm{vb}}\right)$

Tit. دص 20

Texte : Mt 1, 1-25 + Magnificat de Lc 1, 46 avec un renvoi à la fin de la lecture du $2^{\mathrm{e}}$

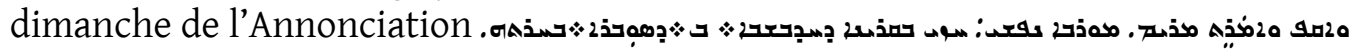

- 10. Deuxième dimanche après la Nativité (f. $8^{\mathrm{vb}}-10^{\mathrm{va}}$ )

Tit.

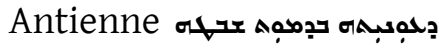

Texte : Lc 2, 21-52.

- 11. Épiphanie (f. $10^{\mathrm{va}}-11^{\mathrm{vb}}$ )

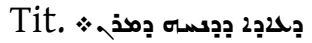

Texte : Mt 2, 21 - 3, 17.

- 12. Commémoraison de saint Jean (Baptiste) (f. $11^{\mathrm{vb}}-12^{\mathrm{vb}}$ )

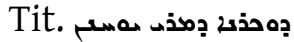

Texte : Mc 6, 14-29 (avec l'addition des derniers mots de $M t 14,12$ ). 
- 13. Premier dimanche après l'Épiphanie (f. $12^{\mathrm{vb}}-14^{\mathrm{ra}}$ )

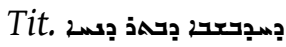

Antienne

Texte : Lc 4, 14-30.

- 14. Commémoraison de Pierre et Paul (f. $14^{\mathrm{ra}}-15^{\mathrm{va}}$ )

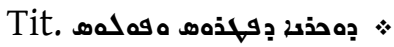

Texte : Mt 16, 13-19+ Jn 21, 15-24.

- 15. Deuxième dimanche après l'Épiphanie (f. $15^{\mathrm{va}}-16^{\mathrm{vb}}$ )

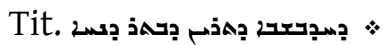

Texte : Jn 1, 1-28 (il manque le tout dernier mot à cause de la lacune).

Lacune de 2 folios après le f. 16. D'après le ms. Cambridge, Add. 1975, et le calcul du nombre de lignes correspondantes, on peut supposer avec toute vraisemblance :

[-16. Commémoraison des Évangélistes]

[Mt 9, 35 - 10, 15]

[-17. Troisième dimanche après l'Épiphanie]

[Jn 1, 29-42]

- 18. [Commémoraison de saint Étienne] (f. $\left.17^{\mathrm{ra}}-18^{\mathrm{ra}}\right)$

Tit. : devait se trouver immédiatement à la fin du folio précédent manquant.

Texte : Mt 11, 20-30+23, 29-33+37-39.

- 19. Quatrième dimanche après l'Épiphanie (f. $18^{\mathrm{ra}}-19^{\mathrm{ra}}$ )

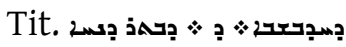

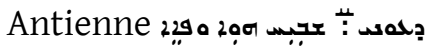

Texte : Jn 1, 43 - 2, 11.

- 20. Commémoraison des docteurs grecs (f. $19^{\mathrm{ra}}-20^{\mathrm{rb}}$ )

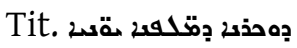

Texte : Mt 4,23 - 5, 19.

- 21. Cinquième dimanche après l'Épiphanie (f. $20^{\mathrm{rb}}-21^{\mathrm{va}}$ )

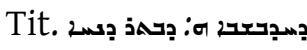

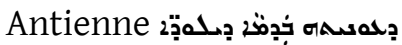

Texte : Jn 2, 23 - 3, 31.

- 22. Commémoraison des docteurs syriens (f. $21^{\mathrm{va}}-22^{\mathrm{va}}$ )

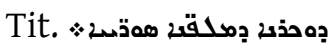

Texte : Mt 16, 24 - 17, 9.

- 23. Sixième dimanche après l'Épiphanie (f. $22^{\mathrm{va}}$ )

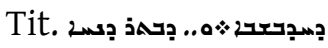

Antienne جهد: جه منبَنسم

Texte : Jn 3, 22-29a.

Lacune de 2 folios après le f. 22 .

- 24. [Commémoraison d'un personnage] (f. ] $23^{\text {ra }}-23^{\text {rb }}$ )

Titre manque.

Texte : Mt [24, 45 - 25, 25a]. 25, 25b-30. 
- 25. Septième dimanche après l'Épiphanie (f. $\left.23^{\mathrm{rb}}-24^{\mathrm{ra}}\right)$

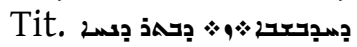

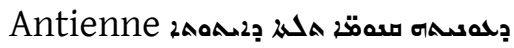

Texte : $M t$ 7, $28-8,13$.

- 28. Commémoraison des défunts (f. $24^{\mathrm{ra}}-25^{\mathrm{ra}}$ )

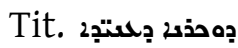

Texte : Mt 25, 31-46.

- 29. Huitième dimanche après l'Épiphanie (f. $\left.25^{\mathrm{ra}}-25^{\mathrm{va}}\right)$

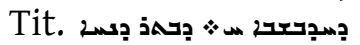

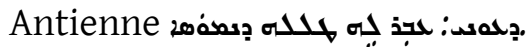

Texte : Mc 1, 1-13.

- 30. Vendredi des 40 martyrs (f. $25^{\mathrm{vb}}$ )

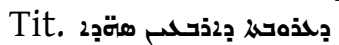

Renvoi à la lecture du vendredi des confesseurs (= vendredi de la semaine sainte)

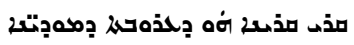

(Texte : $M t$ 10, 16-33)

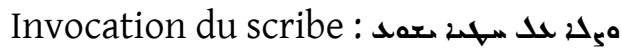

- 31. Dimanche de l'entrée dans le carême de Notre-Seigneur (f. $25^{\mathrm{vb}}-26^{\mathrm{va}}$ )

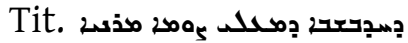

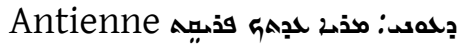

Texte : Mt 3, $16-4,11$.

-32. Lundi de la première semaine du grand carême (f. $26^{\mathrm{va}}-27^{\mathrm{vb}}$ )

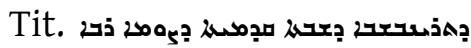

Texte : Mt 5, 17-37.

- 33. Mardi de la première semaine du grand carême (f. $27^{\mathrm{vb}}-28^{\mathrm{rb}}$ )

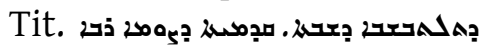

Texte : $M t 5,38-48$.

- 34. Mercredi de la première semaine du grand carême (f. $28^{\mathrm{rb}}-29^{\mathrm{rb}}$ )

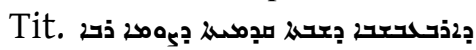

Texte : Mt 6, 1-18.

- 35. Jeudi de la première semaine du grand carême (f. $29^{\mathrm{rb}}-30^{\mathrm{rb}}$ )

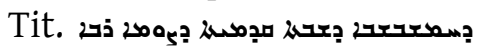

Texte : Mt 6, 19-34.

- 36. Premier Vendredi du grand carême (f. $30^{\mathrm{rb}}-31^{\mathrm{ra}}$ )

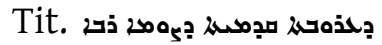

Texte : Mt 7, 1-14.

- 37. Deuxième dimanche du grand carême (f. $31^{\mathrm{ra}}-31^{\mathrm{vb}}$ )

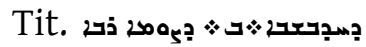

Texte : $M t 7,15-27$.

- 38. Deuxième vendredi du grand carême (f. $31^{\mathrm{vb}}-32^{\mathrm{va}}$ )

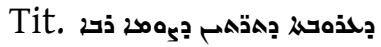

Texte : Mc 11, 27 - 12, 12. 
- 39. Troisième dimanche du grand carême (f. $32^{\mathrm{vab}}[\ldots$...)

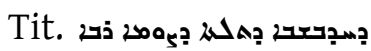

Texte : Mt 20, 17-21a[28.

Lacune d'un folio après le f. 32 : d'après le ms. Cambridge Add. 1975, elle contient la fin de la lecture (jusqu'au verset 28) et le commencement de la lecture suivante ( $M c$ 12, 13-34)

- 40. [Troisième vendredi du grand carême] (f. ...] $33^{\text {ra }}-33^{\text {va }}$ )

Tit. manque.

Texte : Mc 12, [13-]21b-34.

- 41. Quatrième dimanche du grand carême (f. $33^{\mathrm{va}}-35^{\mathrm{ra}}$ )

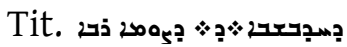

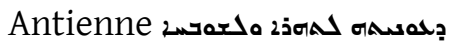

Texte : Mt 21, 23-45.

- 42. Lundi de la semaine de la mi-carême (f. $\left.35^{\text {ra }}-36^{\text {ra }}\right)$

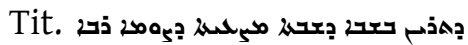

Texte: Jn 5, 1-18.

- 43. Mardi de la semaine de la mi-carême (f. $\left.36^{r a}-37^{v b}\right)$

Tit.

Texte : Jn 5, 19-47.

- 44. Mercredi de la mi-carême (f. $37^{\mathrm{vb}}-39^{\mathrm{ra}}$ )

Tit.

Texte : Jn 6, 51-69.

- 45. Jeudi de la mi-carême (f. $39^{\mathrm{ra}}-39^{\mathrm{vb}}$ )

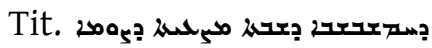

Texte : Jn 7, 1-13.

- 46. Quatrième vendredi de carême (f. $39^{\mathrm{vb}}-40^{\mathrm{vb}}[$ )

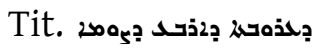

Texte : $J n$ 7, 14-32[-36

Lacune d'1 folio après le f. 40 ; par comparaison avec le ms. Cambridge Add. 1975, elle contient la fin de la lecture 46 et le commencement de la lecture suivante ( $J n$, $37-8,20)$

- 47. Cinquième dimanche de carême (f. $41^{\mathrm{ra}}-41^{\mathrm{va}}$ )

Tit. manque.

Texte : Jn 7, [37-] $50-8,20$.

- 48. Cinquième vendredi de carême (f. $41^{\mathrm{va}}-43^{\mathrm{rb}}$ )

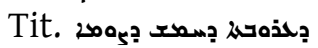

Texte : Jn 8, 31-59.

- 49. Sixième dimanche de carême (f. $43^{\mathrm{rb}}-44^{\mathrm{va}}$ )

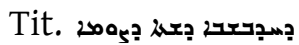

Antienne 21

Texte: Jn 9, 39 - 10, 21. 
- 50. Sixième vendredi de carême (f. $44^{\mathrm{va}}-46^{\mathrm{vb}}$ )

Tit.

Texte : Jn 11, 1-45.

- 51. Septième dimanche de carême dit de Hosana (f. $46^{\mathrm{vb}}-48^{\mathrm{rb}}$ )

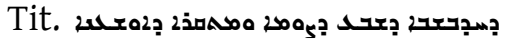

Texte: Mt 20, 29 - 21, 22.

- 52. Lundi de la dernière semaine de carême (f. $48^{\mathrm{rb}}-49^{\mathrm{va}}$ )

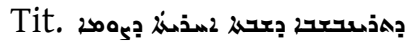

Texte : Jn 11, 47 - 12, 9.

- 53. Mardi de la dernière semaine de carême (f. $49^{\mathrm{va}}-51^{\mathrm{rb}}$ )

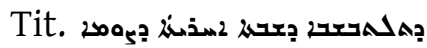

Texte : Jn 12, 12-43.

- 54. Mercredi de la dernière semaine de carême (f. $51^{\mathrm{rb}}-52^{\mathrm{ra}}$ )

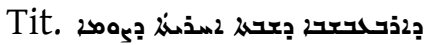

Texte : Jn 13, 1-17.

- 55. Jeudi de la semaine sainte (f. $52^{\mathrm{ra}}-53^{\mathrm{rb}}$ )

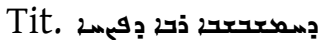

Texte : Mt 26, 1-5.14-30.

- 56. Nuit du vendredi de la Passion (f. $53^{\mathrm{rb}}-55^{\mathrm{vb}}$ )

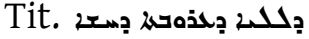

Texte : Mt 26, 31-44 + Lc 22, 43-45 + Mt 26, 45-75.

(f. $56^{\text {ra }}$ blanc)

- 57. Jour du vendredi de la Passion (f. $56^{\mathrm{rb}}-59^{\mathrm{va}}$ )

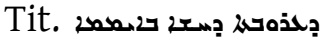

Texte : Lc 22, 63 - 23, 12 ; Mt 27, 19 ; Lc 23, 13- 23 ; Mt 27, 24-25 ; Mt 27, 51-54 ; Jn 19, 23-42; Mt 27, 60b-61; Lc 23, 54-56.

(le modèle du ms. a pu comporter des folios inversés)

- 58. Vêpres du samedi (f. $59^{\mathrm{vab}}$ )

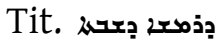

Texte : Mt 27, 62-66.

- 59. Eucharistie du samedi saint $\left(59^{\mathrm{vb}}-60^{\mathrm{vb}}\right)$

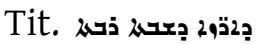

Texte : Mt 28, 1-20.

- 60. Matin de la Résurrection (f. $60^{\mathrm{vb}}-61^{\mathrm{rb}}$ )

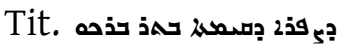

Texte : Lc 24, 1-12.

- 61. Dimanche de la Résurrection (f. $61^{\mathrm{rb}}-62^{\mathrm{va}}$ )

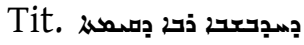

Texte : Jn 20, 1-18.

-62. Lundi de la semaine des semaines (= lundi de Pâques) (f. $62^{\mathrm{va}}-64^{\mathrm{ra}}$ )

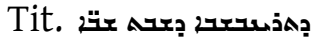

Texte : Jn 14, 18 - 15, 15. 
- 63. Mardi de la semaine des semaines (= mardi de Pâques) $\left(f .64^{r a}-65^{r b}\right)$

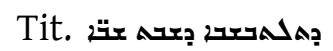

Texte : Lc 24, 13-35.

-64. Mercredi de la semaine des semaines (= mercredi de Pâques) $\left(f .65^{r b}-66^{v a}\right)$

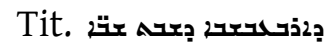

Texte : $J n$ 15, 1-25.

- 65. Jeudi de la semaine des semaines (= jeudi de Pâques) (f. 66 $6^{\mathrm{va}}$ )

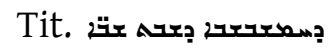

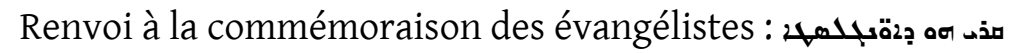

(Texte : Mt 9, 35 - 10, 15).

- 66. Vendredi des confesseurs (f. $66^{\mathrm{va}}-67^{\mathrm{va}}$ )

Tit. بحذهى

Texte : Mt 10, 16-33.

- 67. Dimanche nouveau (octave de Pâques) (f. $67^{\mathrm{va}}-68^{\mathrm{va}}$ )

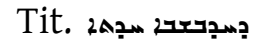

Texte : Jn 20, 19-31.

- 68. Commémoraison de Mar Gīwargīs (f. $68^{\mathrm{va}}-69^{\mathrm{ra}}$ )

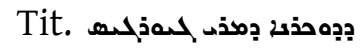

Texte : Mt 10, 37-39 + 19, 27-30.

- 69. Troisième dimanche de la résurrection (f. $69^{\mathrm{rb}}-70^{\mathrm{ra}}$ )

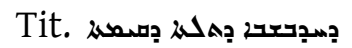

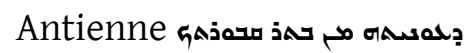

Texte : Jn 14, 1-14.

- 70. Quatrième dimanche de la résurrection (f. $70^{\mathrm{ra}}-71^{\mathrm{ra}}$ )

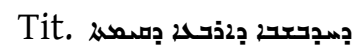

Antienne

Texte : Jn 16, 16-33.

- 71. Cinquième dimanche de la résurrection (f. $71^{\mathrm{ra}}-72^{\mathrm{ra}}$ )

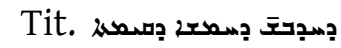

Antienne

Texte : Jn 21, 1-14.

- 72. Sixième dimanche de la résurrection (f. $72^{\text {ra }}-73^{\mathrm{va}}$ )

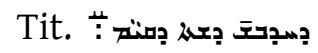

Antienne

Texte : Jn 17, 1-26.

- 73. Fête de l'Ascension de Notre-Seigneur (f. $73^{\mathrm{va}}-74 \mathrm{r}^{\mathrm{b}}$ )

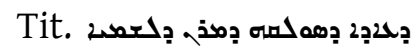

Texte : Lc 24, 36-53.

- 74. Dimanche après l'Ascension (f. $74^{\mathrm{rb}}-75^{\mathrm{rb}}$ )

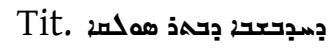

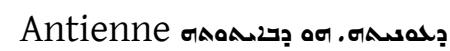

Texte : Mc 16, 1-20. 
- 75. Dimanche de la Pentecôte (f. $75^{\mathrm{rb}}-76^{\mathrm{rb}}$ )

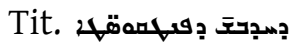

Texte : Jn 14, 15-17.25-26+15, 26 - 16, 15.

- 76. Vendredi d'or $\left(f .76^{r b}-77^{v b}\right)$

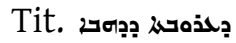

Texte : Lc 7, 1-23.

- 77. Deuxième Dimanche des apôtres (f. $77^{\mathrm{vb}}-79^{\mathrm{ra}}$ )

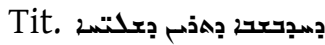

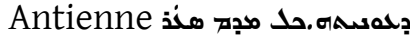

Texte : $L c$ 7, 31-50.

- 78. Troisième dimanche des apôtres (f. $79^{\mathrm{ra}}-80^{\mathrm{ra}}$ )

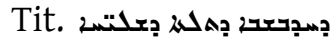

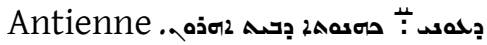

Texte : $L c$ 10, 23-42.

- 79. Quatrième dimanche des apôtres (f. $80^{\text {ra }}-82^{\text {ra }}$ )

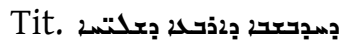

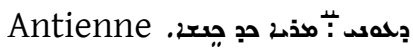

Texte : Lc 6, 12-46.

- 80. Cinquième dimanche des apôtres (f. $82^{\mathrm{ra}}-83^{\mathrm{rb}}$ )

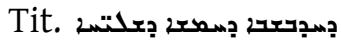

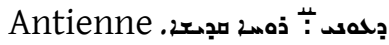

Texte : Lc 12, 16-34.

- 81. Sixième dimanche des apôtres (f. $83^{\mathrm{rb}}-84^{\mathrm{rb}}$ )

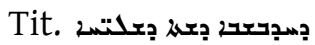

Antienne

Texte : Lc 12, 57 - 13, 17.

- 82. Septième dimanche des apôtres (f. $84^{\mathrm{va}}-85^{\mathrm{rb}}$ )

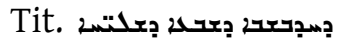

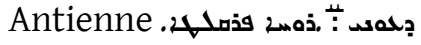

Texte : $L c$ 13, 22-35.

- 83. Dernier vendredi du septénaire des apôtres (f. $85^{\mathrm{rb}}-86^{\mathrm{rb}}$ )

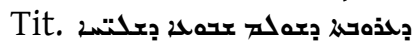

Texte : Mt 10, 37 - 11, 15.

- 84. Dernier dimanche du septénaire des apôtres et premier de l'été (f. $86^{\mathrm{va}}-87^{\mathrm{rb}}$ )

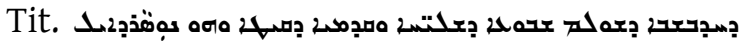

Texte : Lc 14, 1-14.

- 85. Deuxième dimanche de l'été (f. $\left.87^{\text {rb }}-89^{\text {ra }}\right)$

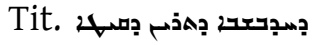

בְם

Texte : Lc 15, 4-32.

- 86. Troisième dimanche de l'été $\left(\right.$ f. $\left.89^{\mathrm{ra}}-91^{\mathrm{ra}}\right)$

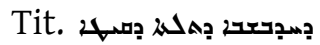

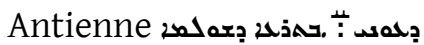

Texte : Jn 9, 1-38. 
- 87. Quatrième dimanche de l'été (f. $91^{\mathrm{ra}}-92^{\mathrm{rb}}$ )

Tit.

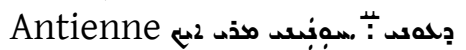

Texte: Mc 7, 1-23 + 7, 16 .

- 88. Cinquième dimanche de l'été (f. $\left.92^{\mathrm{rb}}-93^{\mathrm{vb}}\right)$

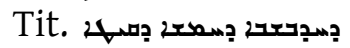

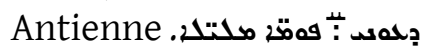

Texte : Lc 16, 19 - 17, 10.

- 89. Sixième dimanche de l'été (f. $93^{\mathrm{vb}}-94^{\mathrm{va}}$ )

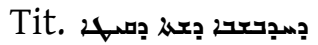

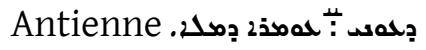

Texte : Lc 17, 5-19.

- 90. Septième dimanche de l'été (f. $94^{\mathrm{va}}$ )

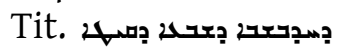

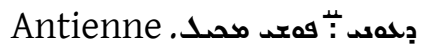

Renvoi au mardi des rogations de Ninive : دمد

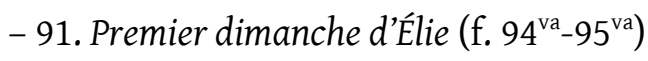

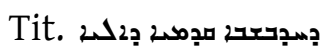

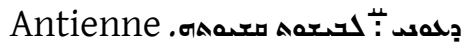

Texte : Lc 18, 35 - 19, 10.

- 92. Deuxième dimanche d'Élie (f. $95^{\mathrm{va}}-96^{\mathrm{vb}}$ )

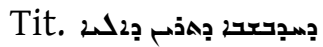

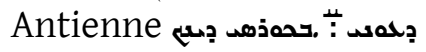

Texte : $M t$ 13, 1-8.

- 93. Troisième dimanche d'Élie (f. $96^{\mathrm{vb}}-98^{\mathrm{ra}}$ )

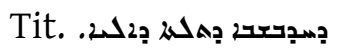

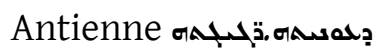

Texte : Mt 13, 24-43.

- 94. Fête de la Croix (f. $98^{\text {ra }}$ )

Tit.

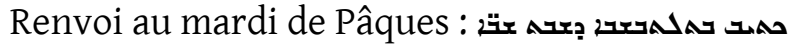

(c'est-à-dire Lc 24, 13-35)

- 95. Quatrième dimanche d'Élie (f. $98^{\text {ra }}-99^{\text {va }}$ )

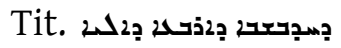

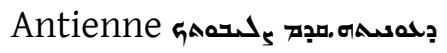

Texte : Mt 4, 12 - 5, 16 .

- 96. Cinquième dimanche d'Élie (f. $99^{\mathrm{va}}-100^{\mathrm{va}}$ )

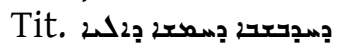

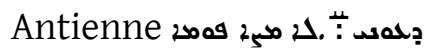

Texte : Mt 17, 14-27.

- 97. Sixième dimanche d'Élie (f. $100^{\mathrm{va}}-101^{\mathrm{va}}$ )

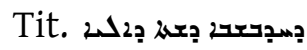




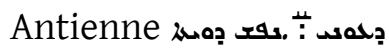

Texte : Mt 15, 21-38.

- 98. Septième dimanche d'Élie (f. $101^{\mathrm{va}}-102^{\mathrm{vb}}$ )

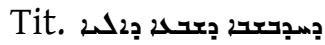

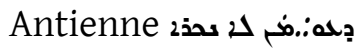

Texte : Mt 18, 1-18.

- 99. Premier dimanche de Moïse (f. $102^{\mathrm{vb}}-103^{\mathrm{va}}$ )

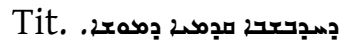

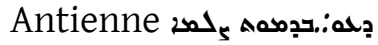

Texte : Mt 20, 1-16+16b $(22,14)$.

- 100. Deuxième dimanche de Moïse (f. $103^{\mathrm{va}}-104^{\mathrm{ra}}$ )

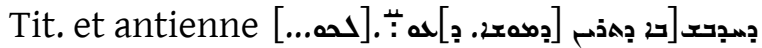

Texte : $L c$ 8, [40-41]42-48. (le début manquant est restitué d'après le ms. Cambridge Add. 1975.)

- 101. Troisième dimanche de Moïse (f. $104^{\mathrm{ra}}$ )

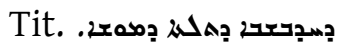

Antienne

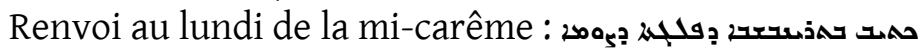

Texte : c'est-à-dire $J n 5,1-18$.

- 102. Quatrième dimanche de Moïse (f. $104^{\mathrm{ra}}-106^{\mathrm{ra}}$ )

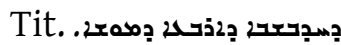

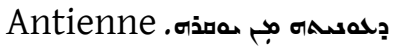

Texte : Jn 4, 1-42.

- 103. Premier dimanche de la consécration de l'église (f. $106^{\mathrm{ra}}-106^{\mathrm{va}}$ )

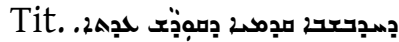

Texte : Mt 16, 13-19+21, 12-13a.

- 104. Deuxième dimanche de la dédicace (de l'église) (f. $106^{\mathrm{va}}-107^{\mathrm{vb}}$ )

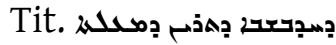

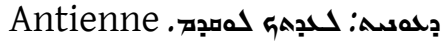

Texte : Mt 12,1-21.

- 105. Troisième dimanche (de la dédicace) de l'église (f. $107^{\mathrm{vb}}-108^{\mathrm{rb}}$ )

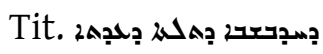

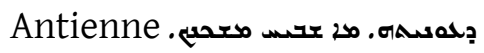

Texte : Jn 2, 12-22.

- 106. Quatrième dimanche (de la dédicace) de l'église (f. $108^{\mathrm{rb}}-109^{\mathrm{va}}$ )

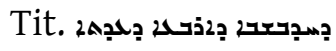

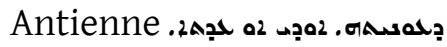

Texte : Mt 22, 41 - 23, 12.16-22.

Expl. du hudrā (f. 109 ${ }^{\text {va }) ~: ~}$

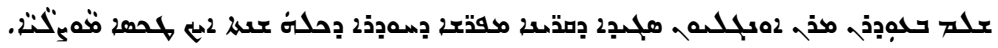

«Fin, avec l'aide de Notre-Seigneur, du saint Évangile des lectures choisies du udra de toute l'année selon l'ordo mossouliote. » 
B. Lectionnaire du gazza (f. $109^{\mathrm{va}}-110^{\mathrm{ra}}$ )

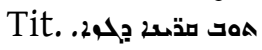

- 1. Pour l'ordination des évêques (f. $109^{\mathrm{va}}$ )

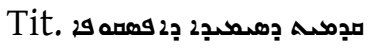

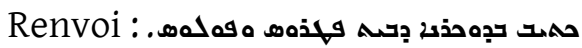

Texte : celui de la commémoraison de Pierre et Paul : Mt 16, 13-19 + Jn 21, 15-24.

- 2. Pour la réception de l'évêque par le catholicos (f. $109^{\mathrm{vb}}$ )

Tit.

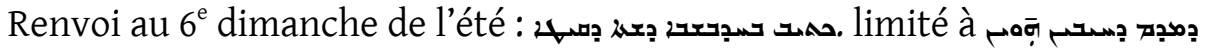

Texte : c'est-à-dire $L c$ 17, 5-10.

- 3. Lorsque l'évêque est ordonné et qu'il monte pour lire (f. $109^{\mathrm{vb}}$ )

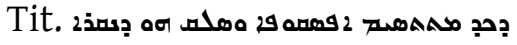

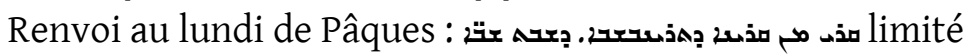

Texte : c'est-à-dire $J n$ 15, 1-15.

- 4. Réception de l'évêque ou du métropolite dans sa région (f. $109^{\mathrm{vb}}$ )

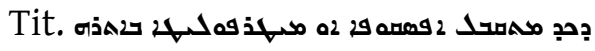

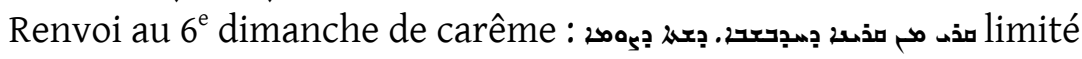

Texte : c'est-à-dire $J n$ 10, 1-16.

- 5. Ordination des diacres (f. $110^{\mathrm{ra}}$ )

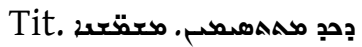

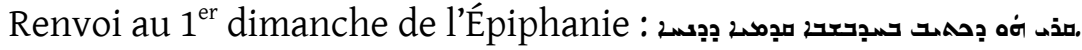

Texte : c'est-à-dire $L c$ 4, 14-30.

C. Lectionnaire des prières pour diverses situations (f. $110^{\mathrm{ra}}-110^{\mathrm{rb}}$ )

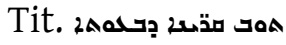

- 1. En cas de manque de pluie (f. $110^{\mathrm{ra}}$ )

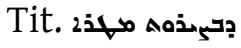

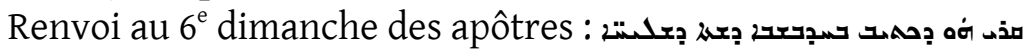

Texte : c'est-à-dire $L c$ 12, 57 - 13, 17.

- 2. En cas de famine (f. $110^{\mathrm{ra}}$ )

בְב 200 حكن

Renvoi au 6 e dimanche d'Élie : مذَ

Texte: c'est-à-dire $M t$ 15, 21-38.

- 3. En cas de tremblement de terre $\left(f .110^{\text {ra }}\right)$

Tit. دمد 200 200

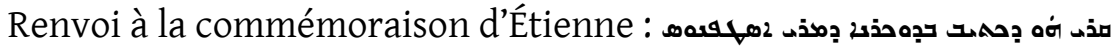

Texte : c'est-à-dire $M t$ 11, 20-30+23, 29-33+37-39.

- 4. Lorsque manquent à l'église la farine et la boisson (f. $110^{\mathrm{rb}}$ )

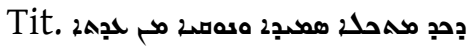

Renvoi au dernier dimanche du septénaire des apôtres : هـ

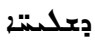

Texte : c'est-à-dire Lc 14, 1-14. 
- 5. En cas de vol des ustensiles liturgiques $\left(f .110^{r b}\right)$

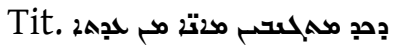

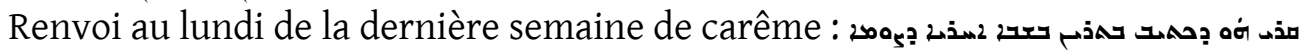

Texte : c'est-à-dire $J n$ 11, 47 - 12, 9.

D. Lectionnaire des prières pour les défunts (f. $110^{\mathrm{rb}}-110^{\mathrm{vb}}$ )

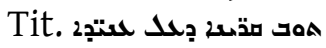

- 1. Pour les catholicoï (f. $110^{\text {rb }}$ )

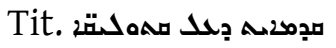

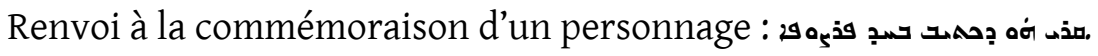

Texte : c'est-à-dire $M t$ 24, 45 - 25, 30.

- 2. Pour le métropolite (f. $110^{\mathrm{rb}}$ )

Tit.

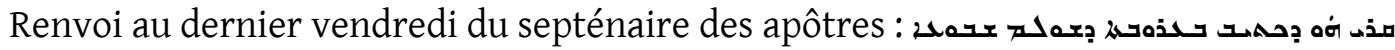

كتلس

Texte : c'est-à-dire $M t$ 10, 37 - 11, 15.

- 3. Pour les évêques (f. $110^{\mathrm{rb}}$ )

Tit.

Renvoi au $2^{\mathrm{e}}$ vendredi du carême : مذّ

Texte : c'est-à-dire $M c$ 11, 27 - 12, 12.

- 4. Pour les docteurs (f. $110^{\mathrm{va}}$ )

Tit. بحد ماكت2

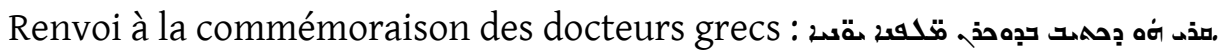

Texte : c'est-à-dire $M t$ 4, 23 - 5, 19.

- 5. Pour les prêtres (f. 110

Tit. بد معتد

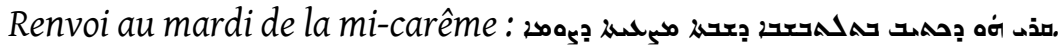

Texte : c'est-à-dire Jn 5, 19-47.

- 6. Pour les moines et les supérieurs de couvents (f. $110^{\mathrm{va}}$ )

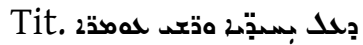

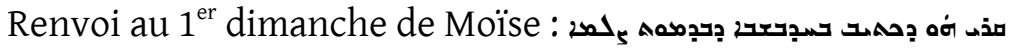

Texte : c'est-à-dire Mt 20, 1-16 + 16b $(22,14)$.

- 7. Pour les diacres (f. $110^{\mathrm{va}}$ )

Tit. בגב مكعتع

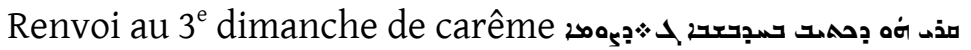

Texte : c'est-à-dire $M t$ 20, 17-28.

- 8. Pour les membres de l'église (f. $110^{\mathrm{va}}$ )

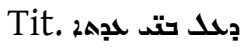

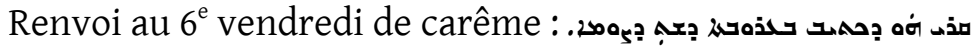

Texte : c'est-à-dire Jn 11, 1-45.

- 9. Pour les fidèles (f. 110 $10^{\mathrm{va}}$ )

Tit. جد متهتع2 


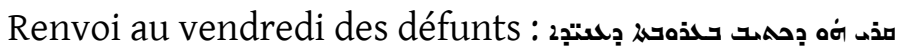

Texte : c'est-à-dire $M t$ 25, 31-46.

- 10. Pour les jeunes gens (f. $110^{\mathrm{va}}$ )

Tit. بد بلدة

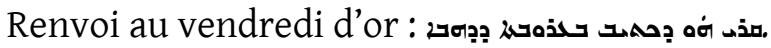

Texte : c'est-à-dire $L c$ 7, 1-23.

- 11. Pour les femmes de l'ordre $\left(110^{\mathrm{vb}}\right)$

Tit. جدا تيم متثخد

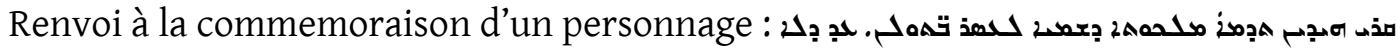

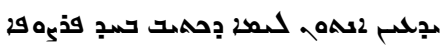

Texte : c'est-à-dire $M t$ 25, 1-13.

- 12. Pour les vieillardes $\left(110^{\mathrm{vb}}\right)$

Tit. بحد بتئ2

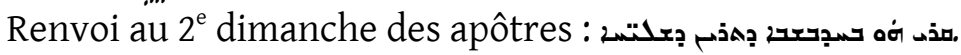

Texte : c'est-à-dire $L c 7,31-50$

- 13. Pour les femmes vertueuses $\left(110^{\mathrm{vb}}\right)$

Tit. בب

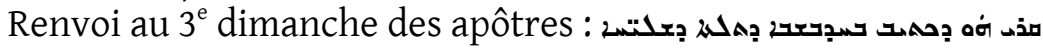

Texte : c'est-à-dire $L c$ 10, 23-42.

- 14. Pour les enfants $\left(110^{\mathrm{vb}}\right)$

Tit.

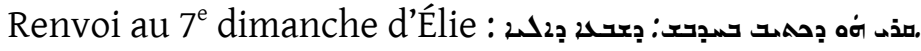

Texte : c'est-à-dire $M t$ 18, 1-18.

Explicit général du manuscrit (f. $\left.110^{\mathrm{vb}}-111^{\mathrm{ra}}\right)$ :

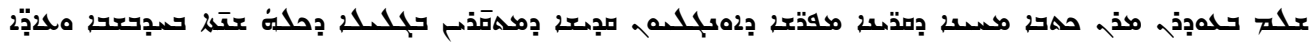

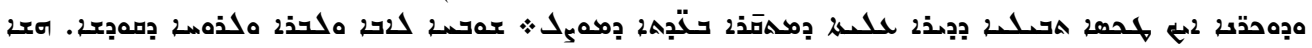

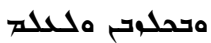

«Est fini avec l'aide de Notre-Seigneur ce vivifiant livre des lectures du saint Évangile, lues toute l'année, pour les dimanches, les fêtes et les mémoires, selon l'ordo universel du couvent supérieur, lu dans les églises de Mossoul. Gloire au Père, au Fils et à l'Esprit-Saint, maintenant et en tout temps. »

Description codicologique :

Support: papier vergé assez épais $(0,22 \mathrm{~mm})$, de couleur blanc sale; trame: 20 vergeures horizontales pour $30 \mathrm{~mm}$, écartement des fils de chaîne : $30 \mathrm{~mm}$, pâte assez homogène, avec parfois quelques concentrations ; filigrane : croix à tête tréflée, dont le bras gauche porte un A (à cheval sur un fil de chaîne) et dont le bras droit porte un Omega vertical dont la courbe inférieure se termine par un petit rond: f. 4, 10, 11, 15, 29, 30, 33, 48, 51, 52, 62, 74, 75, 93, 97, 99, 100, 107 ; trelune horizontal décroissant au f. 112. - encres noire au carbone et cinabre - état de conservation satisfaisant ; la plupart des feuillets sont tachés par une tache brune d'humidité dans la partie inférieure ; au f. 68, une déchirure dans la marge inférieure; le f. 103 est amputé d'une bonne partie de son coin extérieur 
inférieur, qui affecte les deux tiers des col. rb et va ; lacunes : il manque 2 folios avant le f. 1 et après le f. 6 ; il manque 2 folios après le f. 16 et le f. 22 ; il manque 1 folio après le f. 32 et le f. 40 - 112 folios non foliotés ; f. $56^{\text {ra }}, 112^{\text {rb }}$ et $112^{\text {vab }}$ blancs -11 cahiers de 10 folios sauf le cahier XII, de 12 folios; les cahiers I, III et V sont défectueux : au cahier I, il manque les deux premiers bifolios; au cahier III, il manque les 2 premiers bifolios ; au cahier V, il manque le premier bifolio.

Dimensions de la page : $320 \times 220 \mathrm{~mm}$ - mise en pages : 2 colonnes (chacune $245 \times 75$ $\mathrm{mm}$ ), de 20 lignes - interligne: $12 \mathrm{~mm}$ - intercolonnement : $6 \mathrm{~mm}$ - empagement : 245 × $155 \mathrm{~mm}$ - marges : $20 \mathrm{~mm}$ (sup.), 55 (inf.), 25 (int.), 40 (ext.) - pas de réglure visible ; la mise en pages est assurée par un cadre d'empagement formé d'un double filet rouge et l'encadrement de chaque colonne par un filet rouge.

Travail d'écriture: écriture syro-orientale épaisse; hauteur moyenne du module des lettres : 5-6 mm; plus petite lettre : $3 \mathrm{~mm}$; plus grandes lettres : د et a $10 \mathrm{~mm}$ vocalisation systématique par points-voyelles fins - points diacritiques (pronoms affixes, participes actifs) - ponctuation phrastique complexe: pasuqa, šwaye, tahtaye, 'elaye, accent supérieur, accent inférieur, quadruple point en losange de fin de paragraphe justification régulière par allongement des liaisons des dernières lettres et par extension des espaces entre les mots - titres et sous-titres rubriqués - ajouts par le scribe, en marge, de mots oubliés aux f. $7^{\mathrm{r}}, 33^{\mathrm{r}}, 43^{\mathrm{r}}, 70^{\mathrm{r}}, 81^{\mathrm{r}}, 85^{\mathrm{r}}$ - présence de petites lettres interlinéaires (2 et $\Xi)$ au f. $4^{\mathrm{r}}$ sans qu'on perçoive à quoi elles correspondent - décors : un bandeau en tresse noire au f. 25 (marque l'entrée en carême) ; titres en grosses lettres rouges ou noires et en lettres évidées aux f. $6^{\mathrm{v}}$ (Nativité), $10^{\mathrm{v}}$ (Épiphanie), $52^{\mathrm{r}}$ (Pâques) - les signatures de cahiers, par lettres-chiffres syriaques en noir, sont accostées à droite et à gauche d'un nœud de Salomon muni de quatre pointes - marque de folios : le traditionnel signe 3 points / 1 point dans le coin supérieur droit de chaque page - absence de titre courant - aucune réclame.

Reliure syriaque à trois trous d'attache alignés; la couvrure a presque totalement disparu, hormis un lambeau de toile auquel est encore accroché une tranchefile syriaque en trois tresses, brodée bicolore vert et jaune ; ais de bois, d'épaisseur $10 \mathrm{~mm}$; le verso qui avait été cassé à son extrémité sur toute sa longueur a été réparé par des ficelles de serrage ; au recto, quatre petits trous disposés deux par deux sont les traces d'un fermoir ; les contreplats conservent la trace du repli de la couvrure.

Histoire du manuscrit :

Colophon (f. $111^{\mathrm{ra}}-112^{\mathrm{ra}}$ ) :

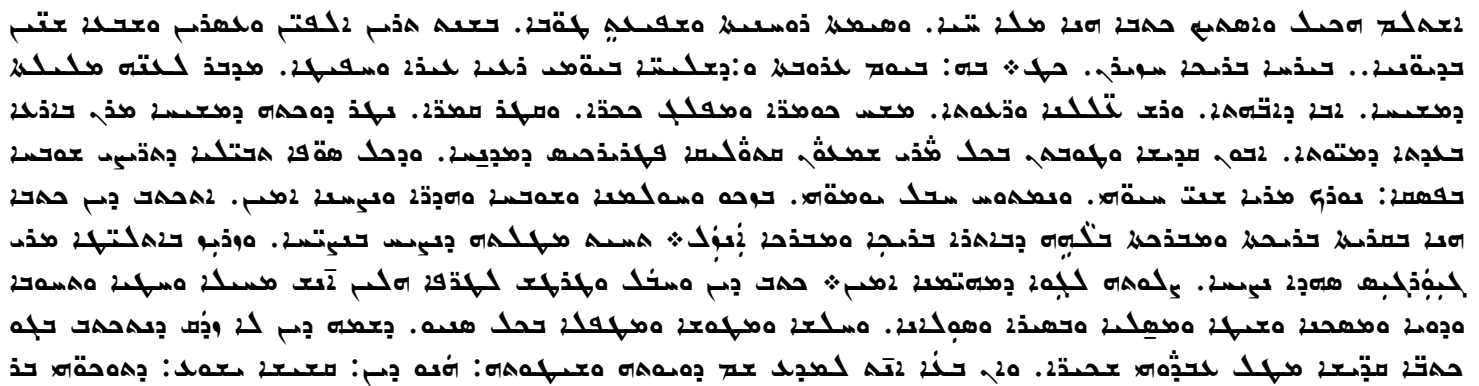




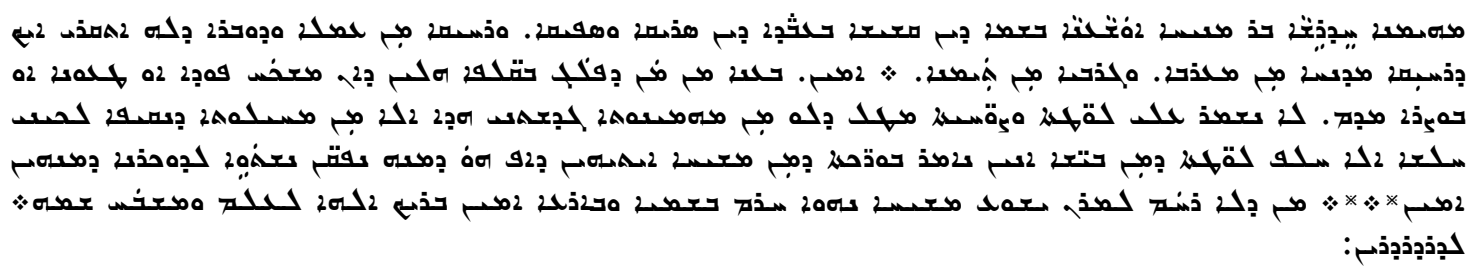

«Ce livre plein de vie, trésor spirituel et d'abondants bienfaits a donc été achevé et terminé en l'an deux mil vingt-sept, année des Grecs, au mois béni de haziran, le 29, le $6^{\mathrm{e}}$ vendredi des apôtres, aux jours du pasteur vigilant et diligent, administrateur du troupeau humain du Christ, père des pères, chef des pasteurs et des bergers, onction des prêtres et répartiteur des talents, nœud des ceintures, gardien du lieu du Christ Notre-Seigneur sur la terre dans l'Église des mortels, notre saint Père et bienheureux en tout Mar Šem 'ōn catholicos patriarche de l'Orient et de tous les confins de la terre habitée des orthodoxes que le Seigneur prolonge les années de sa vie et étende la série de ses jours dans la victoire, la santé, la gloire, la magnificence et l'éclat, Amin.

Ce livre a donc été écrit dans le village béni et digne de bénédiction de B-Lāhēh, dans la région bénie (f. $111^{v}$ ) et digne de bénédiction d'Anzal. Sous l'abri de l'éclatant parmi les éclatants et vaillant parmi les athlètes, Mar Gīwargīs, l'éclatant martyr - sa prière soit au sein des fidèles. Amin.

A écrit, corrompu et taché ces pages, un homme faible, pécheur, infirme, misérable, pauvre, indigne, exécrable, vil, abominable, infortuné, souillé, pollué de toute laideur, dont le nom n'est pas digne d'être écrit dans les livres saints à cause de ses œuvres déshonorantes. Si tu demandes à connaître le nom de sa misère et de son indignité, le voici donc : le prêtre Išo misérable fils du fidèle Hedrēšā fils de feu Ōšānnā, prêtre de nom donc, mais en œuvre, démuni et vain, loin de l'ascèse et de la conduite vertueuse à laquelle il a été appelé, comme est éloigné l'orient de l'occident et le septentrion du midi. Amin.

Je prie celui qui partage ces pages, s'il trouve une faute, une erreur ou un défaut quelconque, qu'il ne m'en accable pas de malédictions ni d'invectives, puisque ce n'est pas par négligence que je les ai commis, mais par la faiblesse inhérente à ma nature fragile ; au lieu des malédictions provenant des mauvais, qu'il dise les bénédictions provenant du Christ, car de Lui elles dérivent ; que nous soyons dignes de mémoire à cause d'elles. Amin .

Celui qui n'aime pas Notre-Seigneur Jésus-Christ, qu'il soit anathème au ciel et sur terre. Amin. Béni soit Dieu à jamais et béni soit son nom, pour les générations des générations. »

L'histoire du manuscrit peut donc se résumer de la façon suivante :

D'après le colophon (f. $110^{\mathrm{vb}}-112^{\mathrm{ra}}$ ) et l'invocation du scribe (f. $25^{\mathrm{vb}}$ ), sa copie a été achevée le vendredi 29 du mois de haziran 2027 selon l'ère séleucide, c'est-à-dire le vendredi 29 juin 1716, qui est le $6^{\mathrm{e}}$ vendredi du cycle liturgique dit " des apôtres », par le prêtre Išō fils du fidèle Hedrēšā, fils de feu Ōšānnā, dans le couvent nestorien Mār-Gīwargīs, dans le village de B-Lāhēh, dans la région d'Anzal, au temps du patriarche catholicos mār Šem ōn, donc le patriarche nestorien de Qočanes, mār Šem`ōn XIV Salomon (1700-1740, selon FIEY 
1993, p. 39 ; voir aussi MurRe-van den Berg 1999 ; Wilmshurst 2000, p. 353). Du temps de ce personnage qui semble faire l'admiration des scribes, a été copié un autre livre liturgique se référant explicitement au rituel ancien du couvent supérieur. En effet, HARRAK 2010 indique que c'est le patriarche sous lequel a été écrit en $1724 \mathrm{AD}$, le šlih $\bar{a}$ du monastère Mār 'Abdišō l'ascète près de Bēth Azzīzā dans le Hakkarī, aujourd'hui ms. Bagdad, Département des Antiquités 30518 dont le colophon, d'ailleurs, parle du patriarche en des termes aussi dithyrambiques que ceux de notre manuscrit.

D'après les notes de propriété en soureth $\left(\mathrm{f} .1^{\mathrm{r}}\right)$, il a servi de livre liturgique en l'église Mart-Mariam de Baznāyā (Turquie).

Notes de lecture modernes griffonnées au f. $69^{v}$.

Remarques sur le texte :

Le rite liturgique auquel se réfère le texte est celui du couvent Mar-Gabriel-et-MarAbraham ou couvent Supérieur (en arabe, Dayr al-ala) de Mossoul, anciennement à l'emplacement de ce qui est aujourd'hui l'église Țāhra des Chaldéens. Amir Harrak note, dans son catalogue ${ }^{6}$ que le patriarche Ǐšocyahb III, réformateur de la liturgie au viI siècle, a pu y vivre. En tout cas, ce rite servit de référence à toute l'Église nestorienne (voir FIEY 1959, p. 126) et, toujours selon Amir Harrak, la mention dans des livres liturgiques récents, du rite du couvent Supérieur qui a disparu au xiII siècle, signifie que ces livres sont des copies d'anciens rituels. On en a plusieurs témoignages, des manuscrits se situant explicitement sous ce patronage liturgique ancien. Le plus ancien connu actuellement semble le šlih $\bar{a}$ conservé à Bagdad, Département des Antiquités, n 30520, antérieur à $1690 \mathrm{AG}$, soit 1378/9 $\mathrm{AD}$ (HARRAK à paraître). J'ai repéré le lectionnaire des évangiles des dimanches et fêtes du ms. London, Rich. 7177, f. 319 daté 1795 AG, soit 1484 AD à Mosul ${ }^{7}$ et le lectionnaire des évangiles des dimanches et fêtes du ms. London, Rich. 7174, f. $212^{v}$ daté 1810 AG, soit 1499 $\mathrm{AD}^{8}$.

Cette référence demeure pour des livres liturgiques postérieurs à la réforme dite d'Alqosh du catholicos Simon VI qui siégea de 1504 à $1538^{9}$; c'est le cas :

- du rituel des dimanches et fêtes du ms. London, Rich. 7178, f. 465 daté 1856 AG, soit $1545 \mathrm{AD}$ à Gozartha (catalogue ForshaLl, no XXXIV, p. 56-57).

- du hudrā du ms. Cambridge, University Library, Add. 1975 daté 1897 AG, soit 1586 $\mathrm{AD}$ à Wastāa dans le jabal Jūdī ${ }^{10}$, et vraisemblablement le hudrā du ms. London, Rich. 7173, f. $1^{\mathrm{v} 11}$.

6. HarRak, Catalogue..., ms. 30518, commentaire sur le f. 69r.

7. Catalogue Forshall 1838, no XXXIII, p. 55-56.

8. Catalogue Forshall 1838, no XXX, p. 51-53.

9. Voir Fiey, 1963, p. 32-33.

10. Catalogue Wright \& Cook 1901, p. 58-80.

11. Catalogue Forshall 1838, no XXIX, p. 48-51. 
- du hudrā du ms. Cambridge, Add. 1981, copié en 1918 AG, soit 1607 AD au couvent Mār-Awdišō' l'ascète à Dere près de Kōm ${ }^{12}$.

- du missel sacerdotal du ms. Cambridge Add. 2045 copié en 1997 AG, soit 1685/6 AD à Māwānā en Tergawar et achevé à Gogtapa ${ }^{13}$.

- du lectionnaire de l'Ancien Testament du ms. Cambridge Add. 1976, copié en 2013 $\mathrm{AG}$, soit $1701 \mathrm{AD}$ à Alqoš ${ }^{14}$.

- du šlīhā et le gazzā du ms. Bagdad 30542, copié en 2128 AG, soit 1719 AD par le diacre Rūbīl de Miniāneš.

- du gazzā du ms. Cambridge, Add. 1980, copié en 2034 AG, soit 1723 AD à Alqoš ${ }^{15}$.

- du šlīhā du ms. Bagdad, Département des Antiquités 30518, copié en 2036 AG, soit 1725 AD.

- du 'annīdā du ms. Cambridge, Add. 1986 copié en 2070 AG, soit 1759 AD à Alqoš ${ }^{16}$.

- du kaškūl du ms. Bagdad, Département des Antiquités 30451, daté 2096 AD, soit 1784 $\mathrm{AD}$, copié également à Miniāneš par le prêtre Hुōšābā.

- du folio étranger du hudrā d'Alqoš, daté 2083 AG, soit 1792 AD au couvent Mar-'Abdišō l'ascète dans la région de Ṭāl, conservé dans le ms. Bagdad, Département des Antiquités 30518.

- du 'annīdā du ms. Bagdad, Département des Antiquités 30527, datable du xviII siècle.

- du šlīhā du ms. Cambridge Add. $2035^{17}$, copié en 2114 AG, soit 1803 AD à Yangyjā près d'Urmia.

- du recueil de turgāmē avant l'évangile pour les fêtes et les commémoraisons, du ms. Bagdad, Département des Antiquités 30525, copié en 2211 AG, soit 1900 AD à Minianeš.

Le ms. Mikaël est un témoignage de plus de cette nouvelle série. Il a un contenu semblable à celui du ms. de Cambridge, University Library, Add. 1975, par rapport auquel il présente quelques variantes dans le contenu des lectures, sans doute par adaptation aux besoins liturgiques de l'église Mar-Georges de B-Lāhēh pour laquelle il a été écrit.

En tout cas, ce type de lectionnaires des évangiles confirme la catégorie de livres liturgiques établie par Fiey (FIEY, 1963, p.26), présentant des calendriers résumés dans lesquels, à la différence de lectionnaires comme par exemple le hudrā de Cambridge Add. 1981 copié en 1607 AD (qui comporte de nombreuses commémoraisons mobiles placées les vendredis et plusieurs fêtes fixes), les commémoraisons ne sont plus portées en dehors de ce que Fiey nomme « les fêtes de base ». Celles-ci sont, dans le lectionnaire de B-Lāhēh, les fêtes de Mart Mariam, Jean-Baptiste, Pierre et Paul, les Évangélistes, Étienne, les Docteurs grecs, les Docteurs syriens, le Patron local (rubrique "Un personnage»), les Défunts, Georges, la Croix, toutes fêtes demeurant à la même place que dans les calendriers les plus

\footnotetext{
12. Catalogue Wright \& Cook 1901, p. 163-193.

13. Catalogue Wright \& CooK 1901, p. 1173-1177.

14. Catalogue Wright \& CoOK 1901, p. 81-107.

15. Catalogue Wright \& Cook 1901, p. 147-163.

16. Catalogue Wright \& Соок 1901, p. 306-309.

17. Catalogue Wright \& CooK 1901, p. 1138-1148.
} 
anciens. C'est le calendrier « résumé » du lectionnaire de l'Ancien Testament et des épîtres dans le ms. Cambridge 1976, daté 1701 AD ; le šliha du ms. Cambridge 2035, daté de 1802/3 AD suit encore le même calendrier. Celui-ci transparaît dans le gazzā du ms. Cambridge 1980, daté de 1723 à Alqōš, qui donne les mawtbē et 'ūnyātā pour les mêmes fêtes « de base » (et en ajoute deux seulement). Cette catégorie de livre liturgique semble donc majoritaire.

Dans le ms. de B-Lāhēh, deux fêtes ont été introduites à ce rang de "fêtes de base »: celle de Bawaï de Nisibe, Jean et leurs compagnons, au $1^{\text {er }}$ vendredi de l'Annonciation, et celle des Quarante martyrs (de Sébaste), placée au $9^{\mathrm{e}}$ vendredi après l'Épiphanie (et non à sa date fixe du 9 mars dans les calendriers récents). Il reste à déterminer à quel moment ces fêtes ont été introduites dans le sanctoral et pourquoi le lectionnaire de B-Lāhēh leur accorde une telle place.

\section{RÉFÉRENCE}

Cassingena-Trévedy (François) \& JuRasz (Izabela), 2006, Les liturgies syriaques, (Études syriaques 3), Paris, Geuthner.

DARMo (Mar Tuma), 1960, métropolite du Malabar, Ktābā dakdām wadbātar wadhudrā wadkaškol wa dgazzā uqālā d'udrānē 'am ktābā dmazmorēe, Trichur, Kérala, Mar Narsai Press, 1993².

ForShAll (J.), 1838, Catalogus codicum manuscriptorum orientalium qui in museo britannico asservantur, Pars prima, Codices syriacos et carshunicos amplectens, London, British Museum .

FIEY (Jean Maurice), 1959, Mossoul chrétienne. Essai sur l'histoire, l'archéologie et l'état actuel des monuments chrétiens de la ville de Mossoul, (Recherches publiées sous la direction de l'Institut de lettres orientales de Beyrouth, t. XII), Beyrouth, Imprimerie catholique.

FIEY (Jean Maurice), 1963, «Le sanctoral syrien oriental d'après les évangéliaires et bréviaires du xI au XIII ${ }^{\mathrm{e}}$ siècle », L'Orient syrien 29 (vol. VIII, 1), p. 21-54.

FIEY (Jean Maurice), 1993, Pour un Oriens christianus novus, Beyrouth.

HaRRAK (Amir), Catalogue of Syriac and Garshuni Manuscripts Owned by the Iraqi Department of Antiquities and Heritage, à paraître.

MurRe-Van den Berg (Heleen H.L.), 1999, « The Patriarchs of the Church of the East from the Fifteenth to Eighteenth Centuries ", Hugoye 2, 2, July.

RüCKER (Adolf), 1932, «Das Obere Kloster bei Mosul und seine Bedeutung für die Geschichte der ostsyrischen Liturgie », Oriens christianus III/7, p. 180-187.

WRIGHT (William) \& CоOK (Stanley Arthur), 1901, A Catalogue of the Syriac Manuscripts preserved in the Library of the University of Cambridge, Cambridge, University Press.

WilmshuRst (David), 2000, The Ecclesiastical Organisation of the Church of the East 1318-1913 (CSCO 582, Subsidia 104), Leuven, Peeters. 


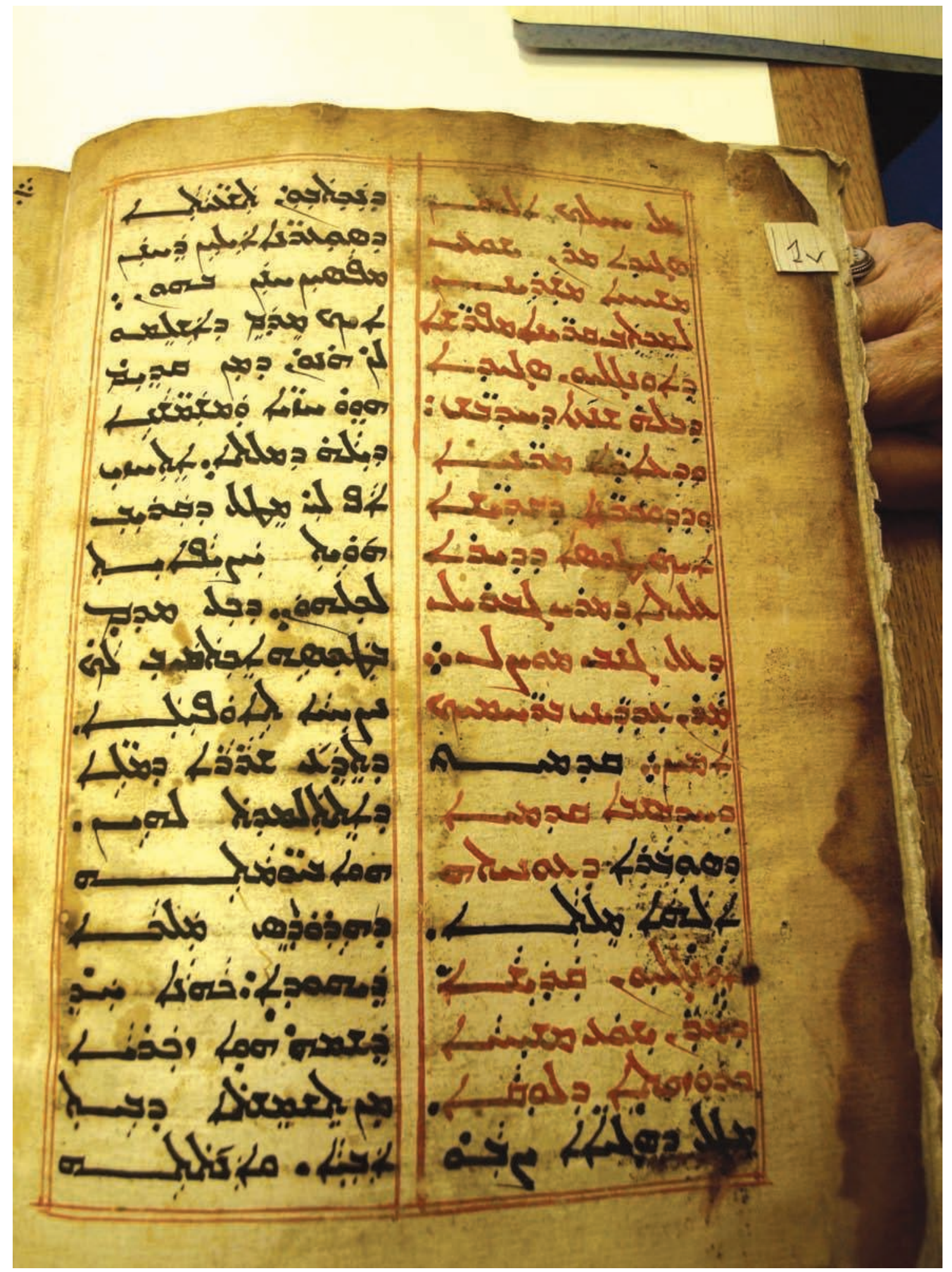




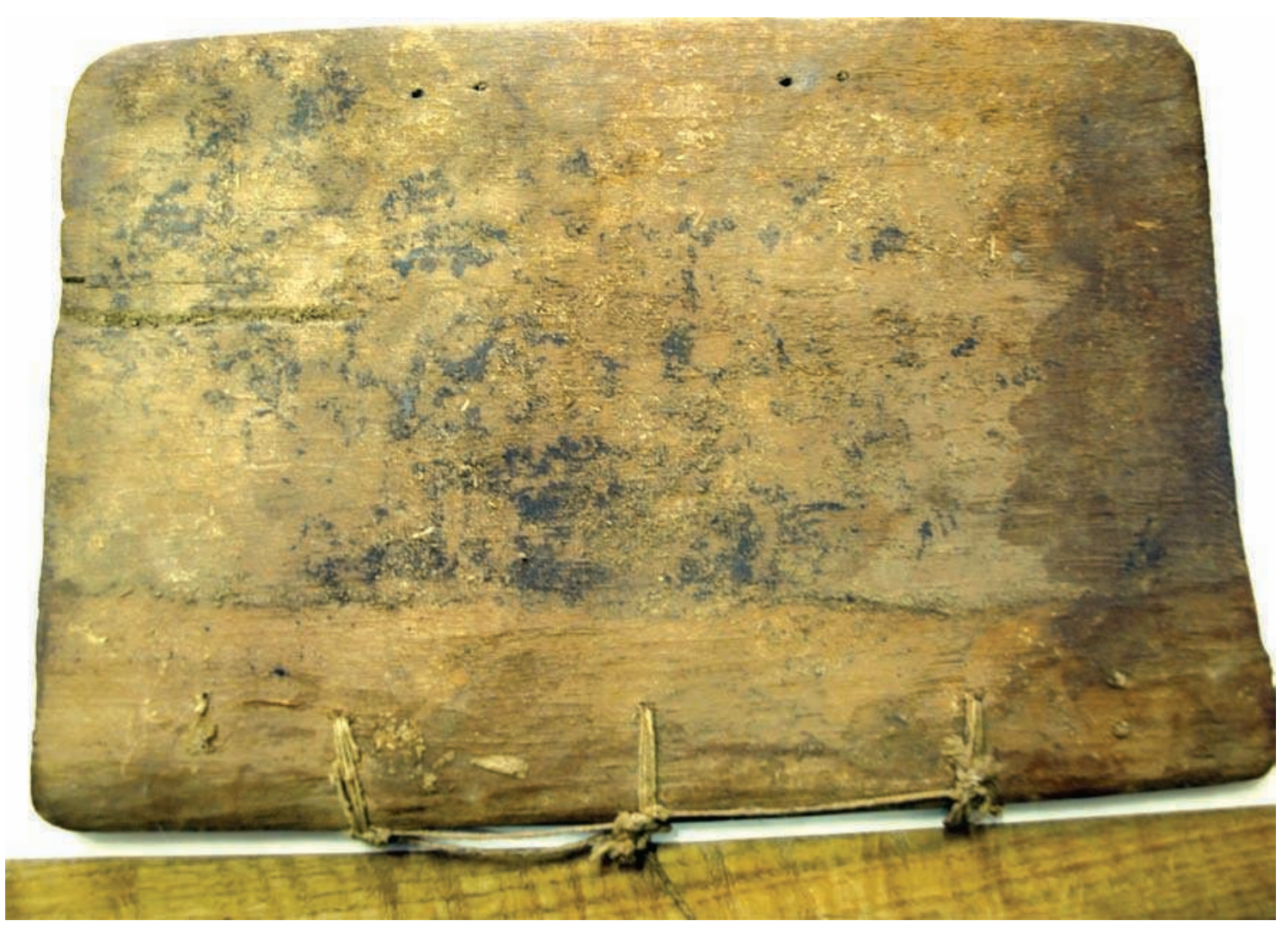




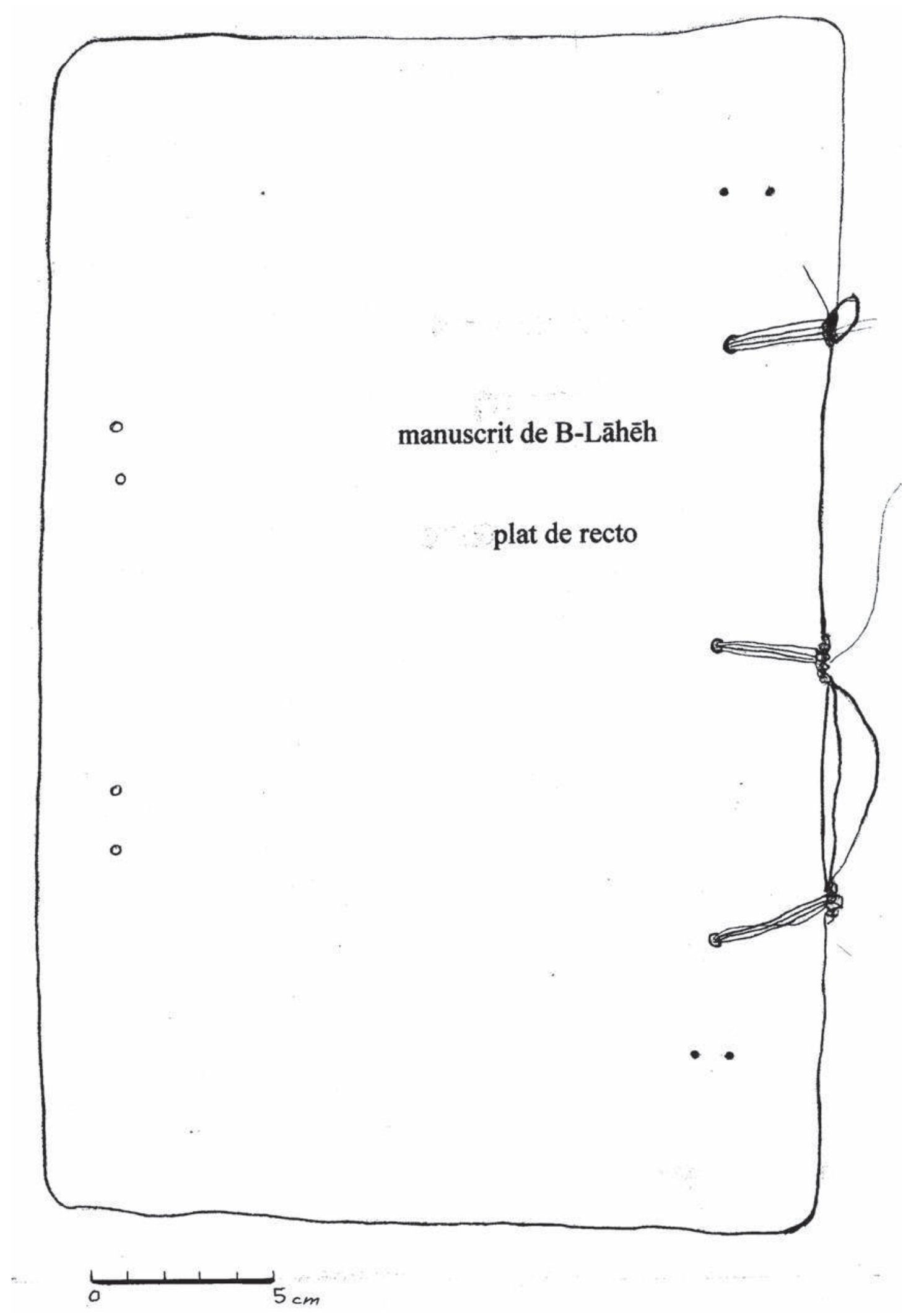




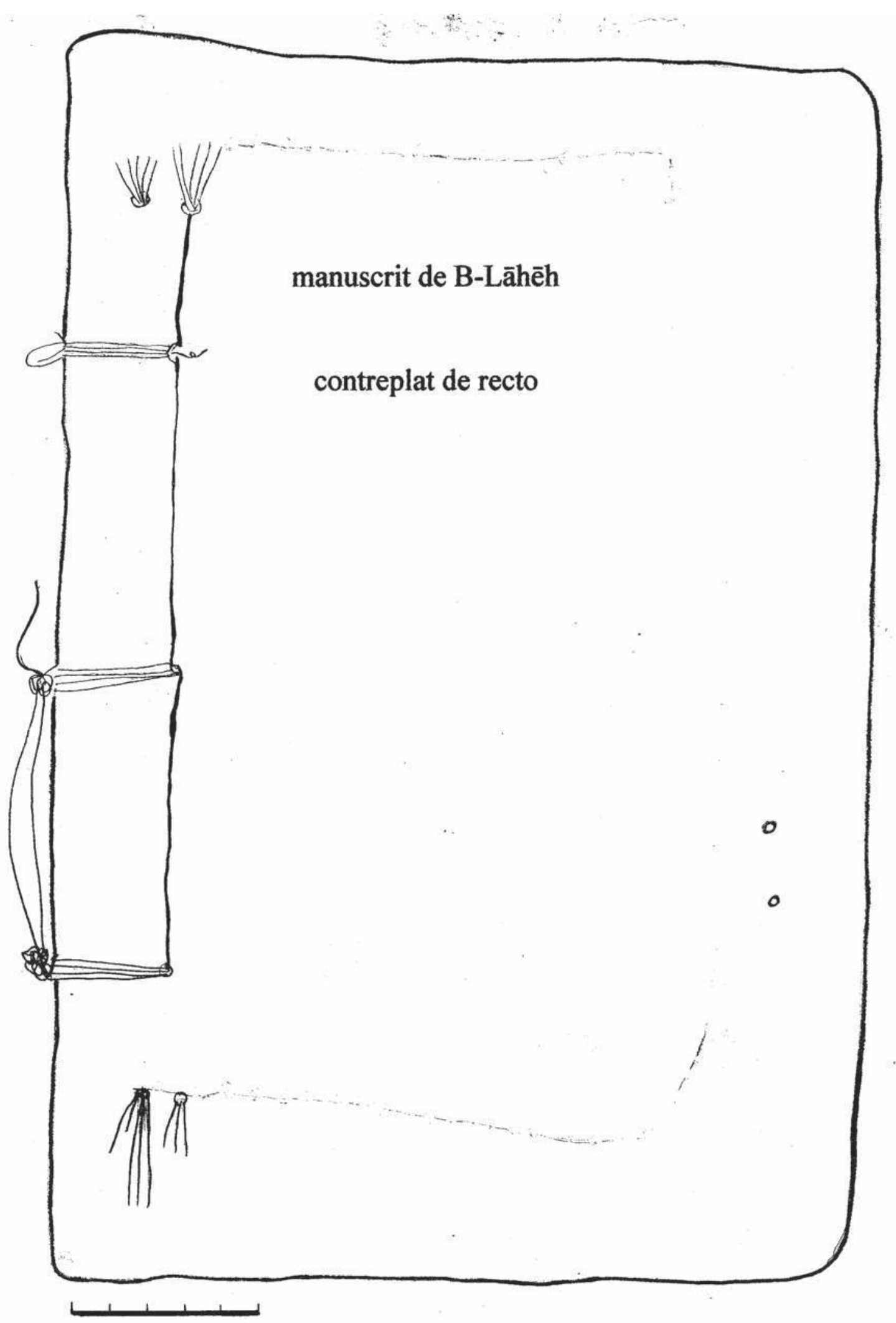




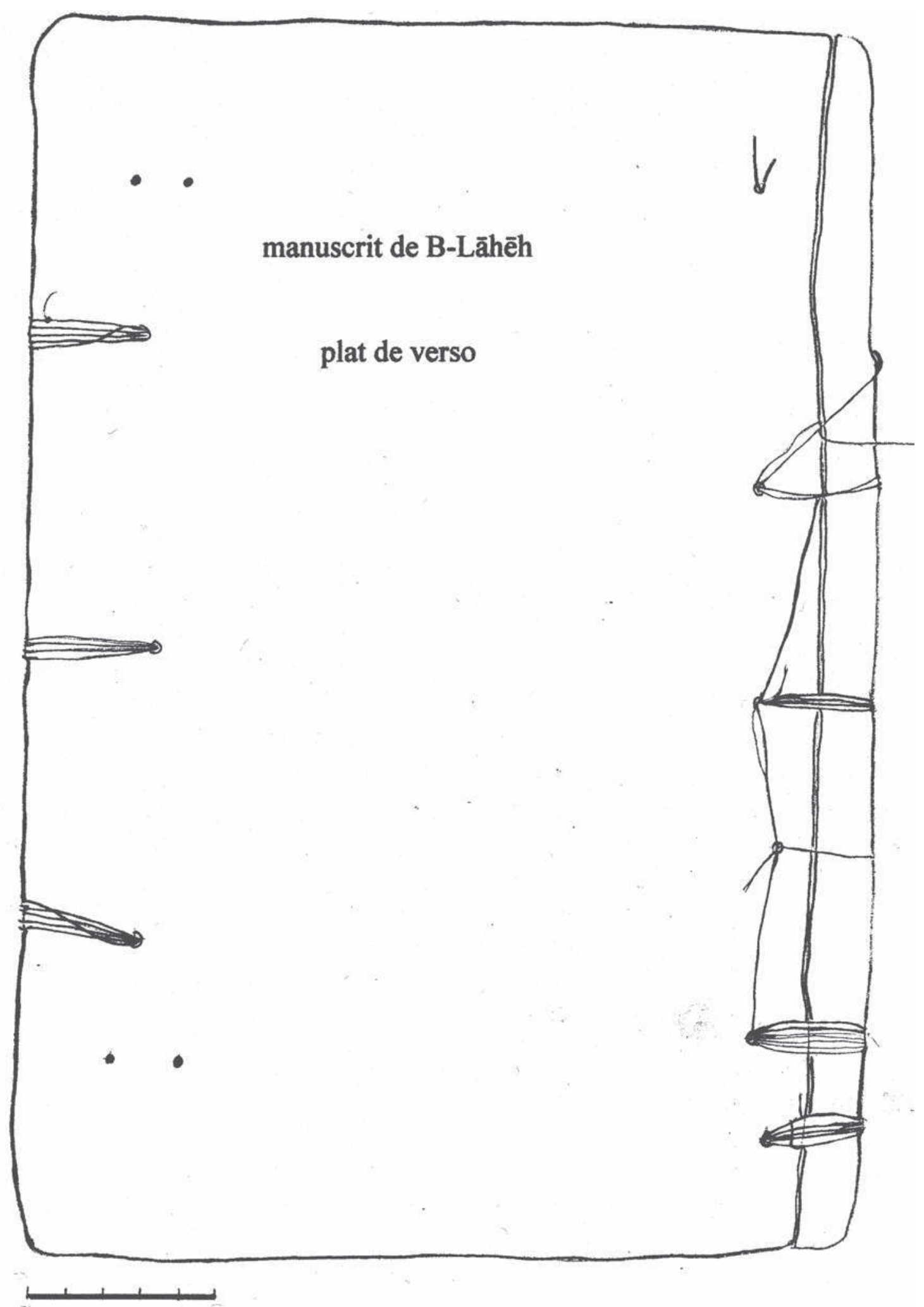




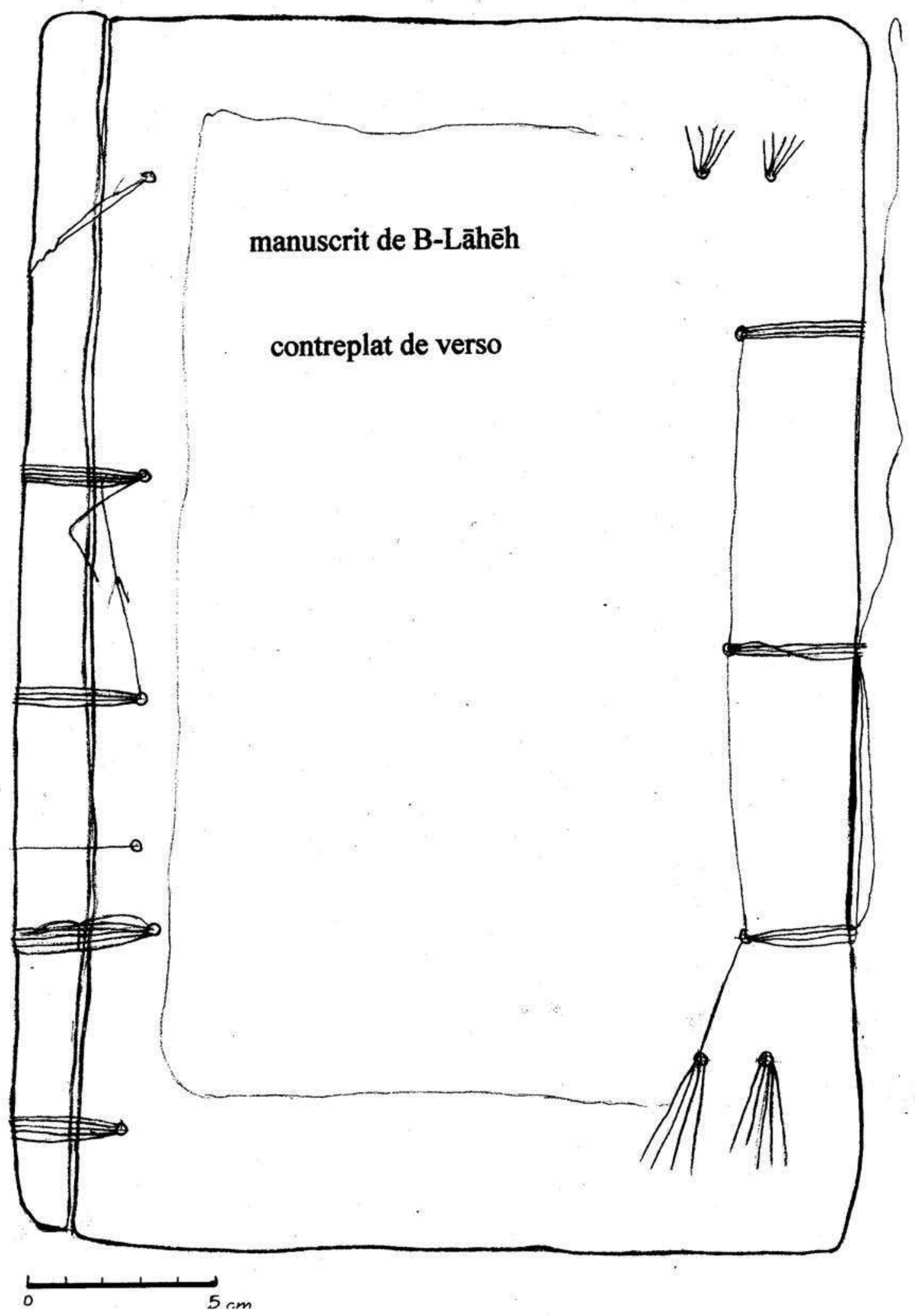

